\title{
Improved brace design combining CAD/CAM and finite element simulation for the conservative treatment of adolescent idiopathic scoliosis (AIS): preliminary results of a randomized control trial
}

Carl-Éric Aubin ${ }^{1,2^{*}}$, Nikita Cobetto ${ }^{1,2}$, Julien Clin ${ }^{1,2}$, Frederique Desbiens-Blais $^{1,2}$, Hubert Labelle $^{2,3}$, Sylvie Le May ${ }^{2,3}$, Stefan Parent ${ }^{2,3}$

From The 10th Meeting of the International Research Society of Spinal Deformities (IRSSD 2014 Sapporo) Sapporo, Japan. 29 June - 2 July 2014

\section{Objectives}

In a previous study on 15 AIS patients, we demonstrated the feasibility of a brace design technology combining Computer Aided Design and Manufacturing (CAD/CAM) and finite element modeling (FEM) for the treatment of scoliosis. The braces showed an equivalent correction to standard braces, but were $61 \%$ thinner and had $32 \%$ less material. A Randomized Control Trial (RCT) was underta-
A)

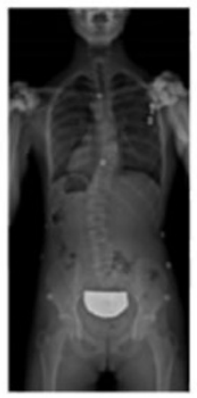

B)
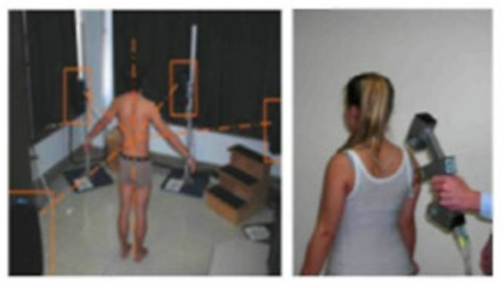

C)
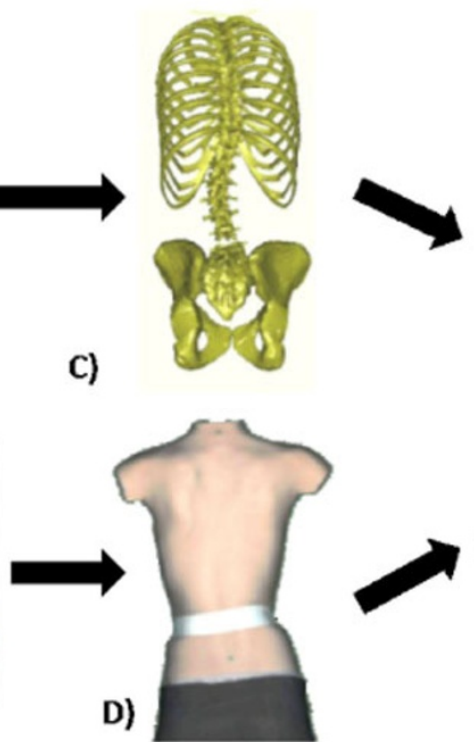
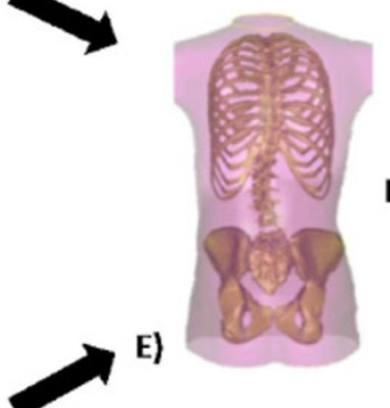

F)

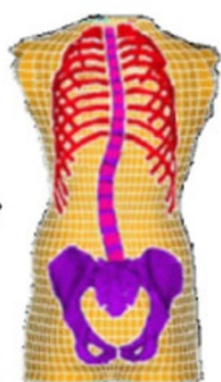

Figure 1 A) Acquisition of the internal geometry using the calibrated bi-planar radiographic 3D reconstruction technique; B) Acquisition of the external geometry using a surface topography system or a scan system; C) Internal 3D geometry; D) External 3D geometry E) Geometric registration F) Finite element model of the trunk

${ }^{1}$ Polytechnique Montreal, Montreal, Canada

Full list of author information is available at the end of the article

(c) 2015 Aubin et al; licensee BioMed Central Ltd. This is an Open Access article distributed under the terms of the Creative Commons 


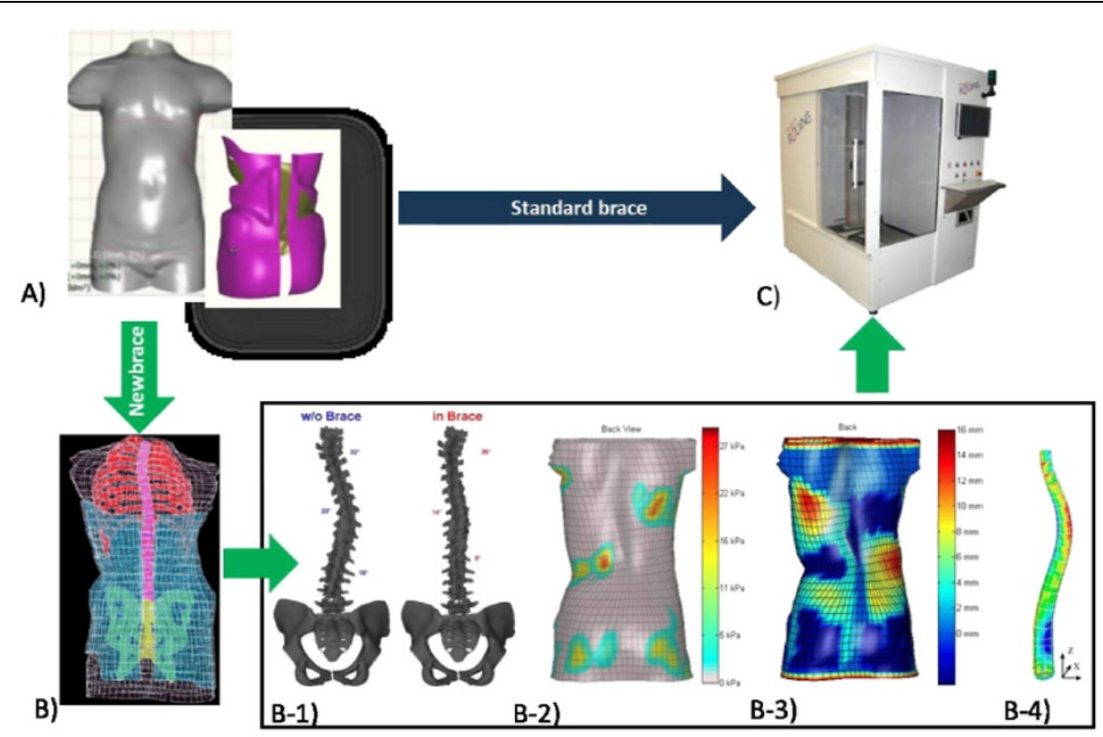

Figure 2 A) Brace design using the CAD software for Standard brace or Newbrace; B) Simulation of the brace installation; B1) Simulation of the spine correction; B2) Simulation of the applied pressures; B3) Simulation of the distance between the brace shell and the patient's skin (the blue color represents the material in contact with the patient's skin and the green, yellow, orange and red colors represent the brace material situated at more than $6 \mathrm{~mm}$ of the patient's skin); B4) Simulated pressures on epiphyseal growth plates; C) Brace fabrication using CAM milling system.

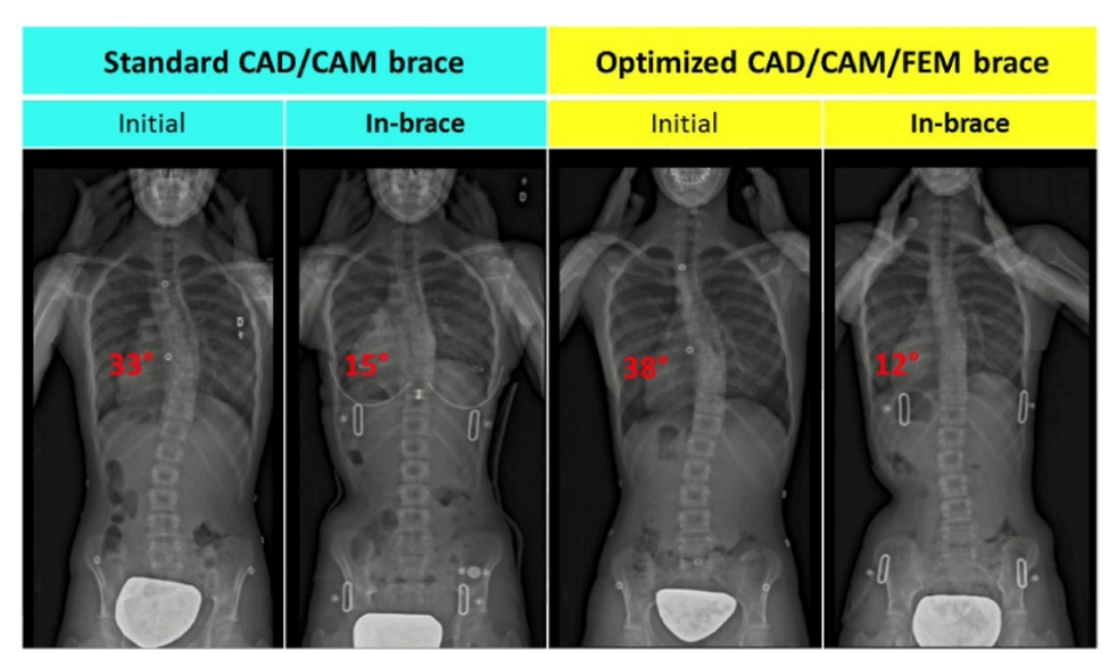

Figure 3 Radiographic results for two typical patients: out of brace for both patients (initial curve), with the StdBrace or with the NewBrace (depending of the randomization), in the postero-anterior view.

ken to pursue the validation and further assess the effectiveness of braces issued from this technology as compared to standard brace design approaches.

\section{Material and methods}

Patients who received a brace treatment prescription were randomized into two groups: 1) TLSO (thoracolumbo-sacral orthosis) fabricated using a scan of patient's torso and a CAD/CAM approach (Rodin4D)
(StandardBrace); 2) TLSO additionally designed using Finite Element Modeling (FEM) built from 3D reconstructions of the trunk skeleton from bi-planar radiographs and optimization (NewBrace). The latter approach allowed to simulate the scoliosis correction and the applied pressures on the torso, and to iteratively design the brace up until the simulated correction was considered maximal and its contact surface with the torso minimal. It was then fabricated using Computer Numerical Controlled technology. 
StandardBrace and NewBrace effectiveness was assessed using radiographs and compared to the simulations.

\section{Preliminary results}

36 patients were consecutively enrolled and to date, 24 patients received their brace. The average Cobb angle prior to bracing was $32^{\circ}$ and $30^{\circ}$ for the main thoracic and lumbar curves respectively. The preliminary results showed that the NewBrace reduced Cobb angles by $47 \%$, which was simulated with a difference inferior to $5^{\circ}$. The StdBrace reduced the Cobb angles by $40 \%$. The NewBrace had $32 \%$ less covering surface than the StdBrace. The highest pressures were located on the thoracic and lumbar regions and at the axillary and the trochanter extensions.

\section{Preliminary conclusion}

So far, a novel design scheme combining CAD/CAM and 3D FEM simulation allowed the fabrication of braces more efficient and lighter than standard TLSO. The study is ongoing to fully compare and validate the effectiveness of the novel design platform.

\section{Consent}

Written informed consent was obtained from the patient for the image(s) used in this study. A copy of the written consent is available for review by the Editor of this journal.

\section{Acknowledgements}

Project funded by the Natural Sciences and Engineering Research Council of Canada, the Canadian Institutes of Health Research, Rodin4D, Boston Brace and Orthèse-Prothèse Rive-Sud.

\section{Authors' details}

${ }^{1}$ Polytechnique Montreal, Montreal, Canada. ${ }^{2}$ Research Center, Sainte-Justine University Hospital Center, Montreal, Canada. ${ }^{3}$ Université de Montréal,

Montreal, Canada.

Published: 19 January 2015

doi:10.1186/1748-7161-10-S1-059

Cite this article as: Aubin et al:: Improved brace design combining CAD/ CAM and finite element simulation for the conservative treatment of adolescent idiopathic scoliosis (AIS): preliminary results of a randomized control trial. Scoliosis 2015 10(Suppl 1):O59.

\section{Submit your next manuscript to BioMed Central} and take full advantage of:

- Convenient online submission

- Thorough peer review

- No space constraints or color figure charges

- Immediate publication on acceptance

- Inclusion in PubMed, CAS, Scopus and Google Scholar

- Research which is freely available for redistribution

Submit your manuscript at www.biomedcentral.com/submit 